\title{
Protein-energy wasting and uremic failure to thrive in children with chronic kidney disease: They are not small adults
}

\author{
Noureddin Nourbakhsh • Connie M. Rhee • \\ Kamyar Kalantar-Zadeh
}

Received: 19 June 2014 / Accepted: 24 June 2014 / Published online: 20 September 2014

(C) IPNA 2014

\begin{abstract}
Protein-energy wasting (PEW), a condition of decreased body protein and fat mass, is highly prevalent in patients with chronic kidney disease (CKD) and a potent predictor of mortality in this population. In adults with CKD, PEW has typically been defined on the basis of (1) deranged biochemical parameters, (2) reduced body mass, (3) reduced muscle mass, and (4) decreased dietary protein intake. Emerging data suggest that PEW may also commonly afflict children with CKD and have a negative impact on growth and development ("uremic failure to thrive"), yet it remains comparatively understudied and less well characterized in these patients. Given the challenges of applying adult-defined PEW criteria to the pediatric population, the authors of a recent study entitled "Protein energy wasting in children with chronic kidney disease" [Abraham et al. (2014) Pediatr Nephrol 29:1231-1238] have sought to develop a scoring system and
\end{abstract}

N. Nourbakhsh $\cdot$ C. M. Rhee $\cdot$ K. Kalantar-Zadeh

Harold Simmons Center, University of California Irvine School of Medicine, Orange, CA, USA

N. Nourbakhsh

Department of Medicine, University of California San Diego School of Medicine, La Jolla, CA, USA

C. M. Rhee $\cdot$ K. Kalantar-Zadeh

Veterans Affairs Long Beach Healthcare System, Long Beach, CA, USA

C. M. Rhee $\cdot$ K. Kalantar-Zadeh

Los Angeles Biomedical Research Institute at Harbor-UCLA

Medical Center, Torrance, CA, USA

K. Kalantar-Zadeh $(\bowtie)$

Harold Simmons Center for Kidney Disease Research and Epidemiology, Division of Nephrology \& Hypertension, University of California Irvine School of Medicine, 101 The City Drive South, City Tower, Orange, CA 92868-3217, USA

e-mail:kkz@uci.edu three alterative definitions for this condition using a combination of biochemical markers, clinical measurements, and subjective reporting in children in the CKiD cohort: (1) minimal PEW definition ( $\geq 2$ adult-defined PEW criteria); (2) standard PEW definition ( $\geq 3$ adult-defined PEW criteria); (3) modified PEW definition ( $\geq 3$ adult-defined PEW criteria, plus short stature or poor growth). These authors observed that meeting the modified PEW definition was associated with a significantly increased risk of hospitalization in unadjusted analyses, i.e., a 2.2-fold higher risk, and trended towards increased risk in multivariable adjusted analyses, i.e., 2.0-fold higher risk. At the present time, future studies validating these findings and developing further refined definitions and/or scoring systems for the detection and management of PEW in children and uremic failure to thrive are urgently needed.

Keywords Protein-energy wasting . Failure to thrive . Cachexia . CKD . Low protein intake - Growth . Development

Protein-energy wasting (PEW) is a common condition afflicting chronic kidney disease (CKD) patients of all ages and is arguably the strongest predictor of mortality, especially in adults and the elderly [1]. Historically, there have been various terms coined by different experts to describe the loss of muscle and fat tissue, malnutrition, and inflammation observed in patients with CKD. For example, uremic malnutrition, uremic (renal) cachexia, protein-energy malnutrition, malnutrition-inflammation atherosclerosis syndrome, and malnutrition-inflammation complex or cachexia syndrome have been frequently utilized, but the term "uremic failure to thrive," abbreviated as uFTT, may be a more appropriate term in the pediatric population given the major impact of CKD on growth and development and the higher risk of cachexia [2]. The term PEW was proposed by the International Society of 
Renal Nutrition and Metabolism (ISRNM; www. RenalNutrition.org) in a consensus statement that has defined this condition as a state of metabolic and nutritional derangement characterized by "decreased body stores of protein and energy fuels (body protein and fat masses)" in both adults and children with CKD [3]. The ISRNM also suggested that the term cachexia be reserved for only the most severe form of PEW. However, the term "uremic failure to thrive" was not mentioned in this consensus statement, nor was attention focused on the specific aspects of PEW in children [3].

Possible causes of PEW include decreased dietary nutrient intake, loss of kidney function, and increase in uremic toxins, dialysis-associated catabolism, anorexia, anemia, metabolic derangements [e.g., hyperparathyroidism, metabolic acidosis, hypogonadism, growth hormone $(\mathrm{GH})$ resistance, vitamin $\mathrm{D}$ deficiency], production of inflammatory cytokines, volume overload, dialysis treatment-related factors (e.g., arteriovenous graft, dialysis membrane), and comorbid conditions (e.g., diabetes, cardiovascular disease, infection, aging). In children, other factors may play additional roles, such as the type of kidney disease (e.g., nephrotic syndrome and autoimmune disorders), psychosocial environment related to the parental role and caregivers, a relative state of GH deficiency, and low protein diet, either prescribed or as a consequence of diminished appetite (see Fig. 1) [4]. The consequences of PEW include cardiovascular disease, infection, depression, increased risk of hospitalization and mortality, as well as failure to thrive in children.

Children with CKD present a unique challenge in the assessment of PEW as the parameters defining this clinical condition in adults [i.e., weight loss, low body mass index (BMI), reduced body fat, reduced muscle mass, inadequate protein intake] are confounded by the normal growth and development of childhood. For instance, BMI in children must be expressed as a percentile or $Z$ score relative to age and sex. Additionally, in the setting of $C K D$, it has been suggested that BMI should be expressed relative to height-age (the age at which the child's height would be at the $50 \%$ percentile), rather than chronological age, due to the high prevalence of growth retardation and delayed sexual maturation [2]. In terms of weight loss, in children, a more appropriate marker of PEW would be failure to gain weight appropriately, as evidenced by a crossing-down of BMI percentiles. Diagnostic criteria for PEW used in adults must either undergo modification for extrapolation to children, or they simply cannot be applied to children due to the lack of data in this population (see Table 1).

The authors of a recent study published in Pediatric Nephrology entitled "Protein energy wasting in children with chronic kidney disease" [5] have sought to combine biochemical markers, clinical measurements, and subjective reporting of children in the Chronic Kidney Disease in Children (CKiD) cohort into a clinically significant scoring system for PEW in

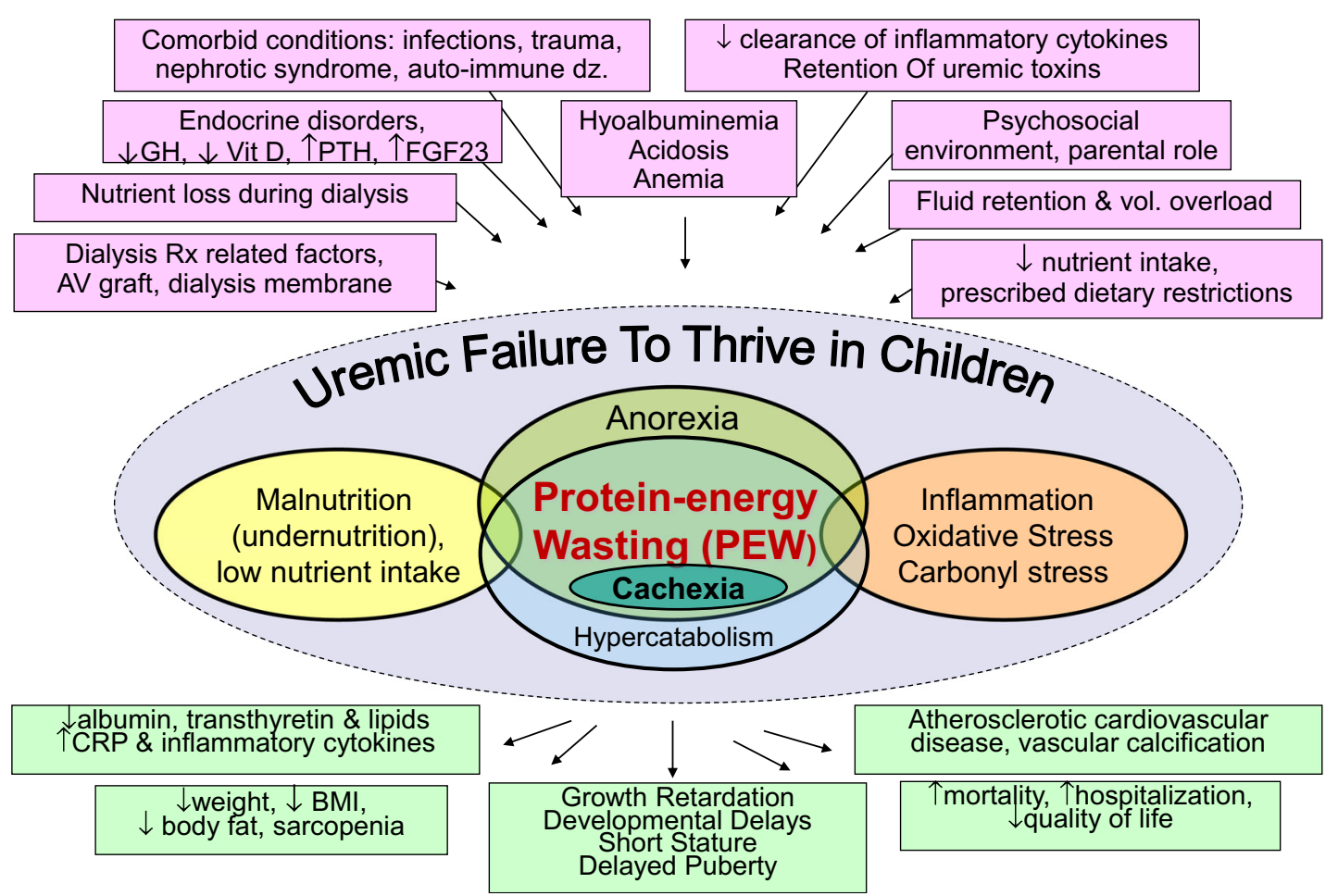

Fig. 1 Schematic representation of pediatric protein-energy wasting $(p P E W)$ and uremic failure to thrive (uFTT) in children with chronic kidney disease. $G H$ Growth hormone, Vit $D$ vitamin $\mathrm{D}, P T H$ parathyroid growth hormone, $F G F 23$ fibroblast growth factor 23, $R x$ renal transplant, $A V$ arteriovenous, $C R P C$-reactive protein, $B M I$ body mass index 
Table 1 Diagnostic criteria for clinical diagnosis of protein-energy wasting in patients with chronic kidney disease, as suggested by and adapted from Abraham et al. [5] (use with permission)

Diagnostic criteria for clinical diagnosis of PEW in patients with CKD

\begin{tabular}{l}
\hline Adult criteria [5] \\
\hline Serum chemistry \\
Serum albumin $<3.8 \mathrm{~g} / 100 \mathrm{~mL}$ \\
(Bromocresol Green) \\
\\
Serum prealbumin \\
(transthyretin) $<30 \mathrm{mg} /$ \\
$100 \mathrm{~mL}$ (for maintenance \\
dialysis patients only; levels \\
may vary according to GFR \\
level for patients with CKD \\
Stages $2-5$ ) \\
Serum cholesterol \\
$<100 \mathrm{mg} / 100 \mathrm{~mL}$
\end{tabular}

\section{Body mass \\ $\mathrm{BMI}<23 \mathrm{~kg} / \mathrm{m}^{2}$}

Unintentional weight loss over time: $5 \%$ over

3 months or $10 \%$ over 6 months

Total body fat percentage $<10 \%$

Muscle mass

Reduced muscle mass $5 \%$ over 3 months or $10 \%$ over 6 months

Reduced mid-arm muscle circumference area (reduction $>10 \%$ in relation to 50th percentile of reference population)

Creatinine appearance

Dietary intake

Unintentional low DPI $<.8 \mathrm{~g} / \mathrm{kg}$ per day for $\geq 2$ months for dialysis patients or $<0.6 \mathrm{~g} / \mathrm{kg}$ per day for patients with CKD stages 2-5

Unintentional low DEI $<25 \mathrm{kcal} / \mathrm{kg}$ per day for $\geq 2$ months

Modified criteria for children [4]

No modification, although specificity of albumin as an indicator of PEW is not assured

No modification although the same issues exist as with albumin

No data exists for association of low cholesterol and outcomes in children with CKD

BMI expressed as percentile or $Z$ score relative to age and sex. Alternatively, BMI for heightage

Failure to gain weight appropriately, as evidenced by crossing of BMI percentiles

Decrease in percentage body fat over time in relation to growth and development

No modification

No modification; although dual energy X-ray absorptiometry is a more accurate marker of muscle mass in children

Not a valid criteria in children as reference values are not available for children

Not evaluated in children as an accurate predictor of PEW

Not evaluated in children as an accurate predictor of PEW

At least three out of the four listed categories (and at least one test in each of the selected category) must be satisfied for the diagnosis of kidney diseaserelated protein-energy wasting (PEW). Optimally, each criterion should be documented on at least three occasions, preferably 2-4 weeks apart.

CKD, Chronic kidney disease; GFR, glomerular filtration rate; BMI, body mass index; DPI, dietary protein intake; DEI, dietary energy intake; PEW children. Their criteria for PEW include: (1) biochemical parameters (total cholesterol $<100 \mathrm{mg} / 100 \mathrm{~mL}$, serum albu$\min <3.8 \mathrm{mg} / \mathrm{dL}$, transferrin $<140 \mathrm{mg} / \mathrm{dL}$, C-reactive protein $<3 \mathrm{mg} / \mathrm{L}$ ); (2) reduced body mass (BMI for height-age of $<5$ th percentile at entry or decrease in BMI for height-age and sex of $>10 \%$ between 1 st and 2 nd annual measurements); (3) reduced muscle mass [mid-upper arm circumference (MUAC) for height-age and sex of $<5$ th percentile or a decrease in MUAC for height-age and sex percentile of $>10 \%$ between the 1 st and 2nd annual visits); (4) decreased appetite as a surrogate for dietary protein intake, reported as fair, poor, or very poor over the week prior to study visit [5]. The scoring system was defined as "minimal PEW" ( $\geq 2$ categories), "standard PEW" ( $\geq 3$ categories), and "modified PEW" ( $\geq 3$ categories), and poor was growth defined as a height for age and sex percentile of $<3$ rd percentile or poor growth velocity or a decrease in height for age of $>10 \%$ between first and second annual visits). The major outcome evaluated was incidence of hospitalization over a 2 -year follow-up period. The authors found a modest association between the modified PEW definition and risk of hospitalization in unadjusted analyses (i.e., 2.2-fold higher risk, unadjusted $p$ value $=0.03$ ), although it did not reach statistical significance in analyses adjusted for confounders (i.e., 2.0-fold higher risk, adjusted $p$ value $=0.06$ ) [5].

An important contribution made by this study is the expansion of the definition of PEW to include growth/growth velocity in children [5]. Without the addition of growth/growth velocity to the scoring system (i.e., the distinction between standard PEW and modified PEW), there would be no statistically significant correlation between PEW and hospitalization risk even in the unadjusted analyses. Although the adjusted analyses did not find a statistically significant association between modified PEW and hospitalization risk, there may be many explanations for this, which we outline below [5].

We believe that refined definitions and/or scoring systems for PEW in children and uremic failure to thrive are urgently needed and that these would serve as better predictors of clinical outcome - specifically in children with CKD — in lieu of criteria currently used to diagnose PEW in adults with CKD. For example, in evaluating the authors' criteria for PEW, based on the CKiD cohort utilized, total cholesterol and transferrin values were the biochemical parameters that showed minimal to no significance in the evaluation of PEW. One could argue that these two values may be abandoned and other markers may be adopted from the adult literature that prove to be of greater significance (e.g., pre-albumin, insulinlike growth factor 1, etc.). It is likely, however, that these other values were not assessed in the CKiD cohort, and for that reason the authors were limited in their evaluation of PEW based on the parameters which were tested.

Also, in evaluating Table 1 of the study [5], which is adopted in our editorial, MUAC as well as BMI (for height, 
age, and sex) showed the most significant contribution to the definition of PEW. Therefore, it may be worthwhile to establish a weighted scoring system to define PEW with a greater emphasis given to low MUAC and BMI. Similar to scoring systems for other clinical entities (e.g. sepsis), there may be benefit in weighting MUAC and BMI over other parameters in the assessment of PEW, and the final weighted score may show more clinical significance than simply an assessment of additive criteria.

Second, in the adult CKD literature, PEW manifests at an advanced stage of kidney disease (either early, before, or during initiation of dialytic therapy) [6,7]. As the authors suggest in their discussion, the CKiD cohort evaluated 528 children with estimated glomerular filtration rate (eGFR) values of between 30 and $90 \mathrm{~mL} / \mathrm{min} / 1.73 \mathrm{~m}^{2}$ (median eGFR $45 \mathrm{~mL} / \mathrm{min} / 1.73 \mathrm{~m}^{2}$ ), and it may be that the statistical significance of the association between PEW and hospitalization would be more robust with a cohort of children with advanced CKD (i.e. CKD Stage 4 to 5). Moreover, if the period of follow-up of these children were extended, there may also be greater value in the authors' definition of PEW and association with hospitalization risk. In future analyses of the CKiD cohort, other parameters beyond hospitalization risk may be evaluated in relation to PEW (i.e. quality of life, mortality, etc) [5]. Furthermore, it is not surprising, as shown in the article [5], that the standard PEW definition had lower prevalence in the CKiD cohort than minimal PEW and modified PEW definitions, as the definition of minimal PEW is broad (only $>2$ categories required) while the definition of modified PEW is more precise ( $>3$ categories, plus poor growth). Also, as shown in the study [5], there was no statistically significant increase in PEW with incrementally higher CKD stage, which argues that either the definition of PEW is poor or there really is not a significant association between early CKD stage (i.e., Stages 1-3) and PEW. Third, the power of the PEW scoring system may be improved by increasing the sample size of the study [5]. Again, as the authors noted in their discussion section, the number of children enrolled in the study cohort may limit the power of the PEW score in predicting hospitalization/outcomes. Finally, the fourth criterion for PEW, as defined by the authors, was a subjective reporting of decreased appetite as a surrogate for dietary protein intake. Although technically more complicated, an actual diary of dietary intake over a defined period would serve as a more accurate measurement of protein intake and likely allow for added statistical significance of the PEW scoring system in correlation to clinical outcome.

In summary, given the decreased dietary nutrient intake, loss of kidney function, and metabolic derangements that accompany advanced CKD, it is of utmost importance to diagnose PEW early in order to maintain dietary protein and energy intake and to avoid complications arising from PEW. In children with $\mathrm{CKD}$, preemptive diagnosis of PEW is further complicated by the unique aspects of pediatric growth and development that are altered in kidney disease. Although the aforementioned study has provided insight into potential measures of PEW, further research in the field of PEW in children with CKD is required before specific criteria are established for this complex diagnosis.

Funding Source The authors are supported by the research grants from the NIH/NIDDK including K24-DK091419 (KKZ), R01-DK078106 (KKZ), and philanthropist grants from Mr. Harold Simmons and Mr. Louis Chang.

\section{Potential Conflicts of Interest None.}

\section{References}

1. Kim JC, Kalantar-Zadeh K, Kopple JD (2013) Frailty and proteinenergy wasting in elderly patients with end stage kidney disease. J Am Soc Nephrol 24:337-351

2. Mak RH, Cheung WW, Zhan JY, Shen Q, Foster BJ (2012) Cachexia and protein-energy wasting in children with chronic kidney disease. Pediatr Nephrol 27:173-181

3. Fouque D, Kalantar-Zadeh K, Kopple J, Cano N, Chauveau P, Cuppari L, Franch H, Guarnieri G, Ikizler TA, Kaysen G, Lindholm B, Massy Z, Mitch W, Pineda E, Stenvinkel P, Trevino-Becerra A, Wanner C (2008) A proposed nomenclature and diagnostic criteria for protein-energy wasting in acute and chronic kidney disease. Kidney Int 73:391-398

4. Kovesdy CP, Kopple JD, Kalantar-Zadeh K (2013) Management of protein-energy wasting in non-dialysis-dependent chronic kidney disease: Reconciling low protein intake with nutritional therapy. Am J Clin Nutr 97:1163-1177

5. Abraham AG, Mak RH, Mitsnefes M, White C, Moxey-Mims M, Warady B, Furth SL (2014) Protein energy wasting in children with chronic kidney disease. Pediatr Nephrol 29:1231-1238

6. Kovesdy CP, George SM, Anderson JE, Kalantar-Zadeh K (2009) Outcome predictability of biomarkers of protein-energy wasting and inflammation in moderate and advanced chronic kidney disease. Am J Clin Nutr 90:407-414

7. Kovesdy CP, Kalantar-Zadeh K (2009) Why is protein-energy wasting associated with mortality in chronic kidney disease? Semin Nephrol 29:3-14 